\title{
ResMem-Net: Memory based deep CNN for image memorability estimation
}

\author{
Arockia Praveen ${ }^{1}$, Abdulfattah Noorwali ${ }^{2}$, Duraimurugan Samiayya ${ }^{3}$, Mohammad Zubair Khan ${ }^{4}$, Durai Raj Vincent \\ P $\mathbf{M}^{\text {Corresp., } 5}$, Ali Kashif Bashir ${ }^{6}$, Vinoth Alagupandi ${ }^{3}$ \\ 1 Phosphene Al, Chennai, India \\ 2 Umm Al-Qura University, Makkah, Saudi Arabia \\ 3 Department of information Technology, St. Joseph's College of Engineering, Chennai, India \\ 4 Taibah University, Madinah, Saudi Arabia \\ 5 School of Information Technology and Engineering, Vellore Institute of Technology University, Vellore, Tamilnadu, India \\ 6 The Manchester Metropolitan University, Manchester, United Kingdom \\ Corresponding Author: Durai Raj Vincent P M \\ Email address: pmvincent@vit.ac.in
}

Image memorability is a very hard problem in Image Processing due to its subjective nature. But due to the introduction of Deep Learning and the large availability of data and GPUs, great strides have been made in predicting the memorability of an image. In this paper, we propose a novel deep learning architecture called ResMem-Net that is a hybrid of LSTM and CNN that uses information from the hidden layers of the CNN to compute the memorability score of an image. The intermediate layers are important for predicting the output because they contain information about the intrinsic properties of the image. The proposed architecture automatically learns visual emotions and saliency, shown by the heatmaps generated using the GradRAM technique. We have also used the heatmaps and results to analyze and answer one of the most important questions in image memorability: "What makes an image memorable?". The model is trained and evaluated using the publicly available Large-scale Image Memorability dataset (LaMem) from MIT. The results show that the model achieves a rank correlation of 0.679 and a mean squared error of 0.011 , which is better than the current state-of-the-art models and is close to human consistency $(p=0.68)$. The proposed architecture also has a significantly low number of parameters compared to the state-of-the-art architecture, making it memory efficient and suitable for production. 
2 ResMem-Net: Memory based Deep CNN for Image 3 Memorability Estimation

4

5

6

7

8

Arockia Praveen J B 1 , Abdulfattah Noorwali2, Duraimurugan $\mathrm{S}^{3}$, Mohammad zubair khan ${ }^{4}, \mathrm{P} \mathrm{M}$ Durai Raj Vincent ${ }^{5}$, Ali Kashif Bashir ${ }^{6}$, Vinoth A $^{3}$

${ }^{1}$ Phosphene Al, Chennai, Tamil Nadu, India.

2 Department of Electrical Engineering, Umm Al-Qura University, Makkah, Saudi Arabia

${ }^{3}$ Department of information Technology, St. Joseph's College of Engineering, OMR, Chennai, Tamil Nadu, India.

${ }^{4}$ Department of computer science, College of computer science and engineering, Taibah University Madinah Saudi Arabia

${ }^{5}$ School of Information Technology and Engineering, VIT Vellore, Tamil Nadu, India.

${ }^{6}$ Department of Computing and Mathematics, Manchester Metropolitan University, UK

Corresponding Author:

P M Durai Raj Vincent

School of Information Technology and Engineering, VIT Vellore, Tamil Nadu, India.

Email address: pmvincent@vit.ac.in 
42

43

44

45

46

47

48

49

50

51

52

53

54

55

56

57

58

59

60

61

62

63

64

65

66

67

68

69

70

71

72

73

74

75

76

77

78

79

80

81

\section{Abstract}

Image memorability is a hard problem in Image Processing due to the problem's subjective nature. But with the introduction of Deep Learning, availability of huge data and GPUs, great strides have been made feasible in predicting the memorability of an image. This paper proposes Residual Memory Network (ResMem-Net), a novel deep learning architecture that is a hybrid of Long Short-Term Memory (LSTM) and Convolutional Neural Networks (CNN). This architecture uses information from the hidden layers of the $\mathrm{CNN}$, which are the learned convolution filters that contain extract features from the input, to compute the memorability score of an image. The intermediate layers are important for predicting the output because they contain information pertaining to the intrinsic properties of the image. The proposed architecture automatically learns visual emotions and saliency, as shown by the heatmaps generated using the Gradient Regression Activation Map (GradRAM) technique. The study also used the heatmaps and results to analyze and answer one of the most important questions in image memorability which is "What makes an image memorable?". The proposed model is trained and evaluated using the publicly available Large-scale Image Memorability dataset (LaMem) from MIT. The result shows that the proposed model achieves a rank correlation of 0.679 and a mean squared error of 0.011 , which is better than the current state-of-the-art models and is close to human consistency $(p=0.68)$. The proposed architecture also has a significantly low number of parameters compared to the state-of-the-art architecture, making it memory efficient and suitable for production.

Keywords: Deep Learning, Image Memorability, Visual Emotions, Saliency, Object Interestingness

\section{Introduction}

Data is core essential component to almost every media platform in this digital era, starting from the television to social networks. Every media platform relies on content to engage their users. It provides a compulsion for these platforms to understand the exponentially growing data to serve the right content to their users. Since most of these platforms rely on visual data, concepts such as popularity, emotions, interestingness, aesthetics, and, most importantly, memorability are very crucial in increasing viewership (Kong et al., 2016; Celikkale, Erdem \& Erdem, 2015). In this paper, image memorability is the concept taken into consideration which is one of the most underexplored deep learning applications.

Human beings normally rely on visual memories to remember things and also will be able to identify and discriminate objects in real life. Human cognition to properly remember and forget visual data is crucial as it affects every form of our engagement with the external world (Bainbridge, Dilks \& Oliva, 2017; Schurgin, 2018). However, not all humans remember the 
82 same visual information in a common manner (Gretz \& Huff, 2020; Rust, Mehrpour., 2020). It is 83 a long-standing question that neuroscientists have asked for years, and research is still underway 84 to explain how exactly the cognitive processes in the brain encode and store certain information 85 to retrieve that information when required properly. The human brain can encode intrinsic 86 information about objects, events, words, and images after a single exposure to visual data 87 (Alves et al., 2020; Fukuda, Vogel., 2019).

88 Image memorability is generally measured as the probability that a person will be able to identify 89 a repeated photograph when he or she is presented with a stream of images (Isola et al., 2011a). 90 By definition, image memorability is a subjective measure that approximately quantifies how a 91 person can remember an image (Isola et al., 2011b). Cognitive psychologists have shown that 92 more memorable images leave a larger trace of the brain's long-term memory (Broers \& Busch , 2021). However, the memorability of a certain image can slightly vary from person to person and

94

95

96

97

98

99

100

101

102

103

104

105

106

107

108

109

110

111

112

113

114

115

116

117

118

119

120

121

depends on the person's context and previous experiences (Bainbridge, 2020). But this slight variation is fine because this allows us to make approximate predictions using computational methods.

Researchers have shown that, even though there exist slight variations, humans show a level of consistency when remembering the same kind of images with a very similar probability irrespective of the time delay (Sommer et al., 2021). This research has led to the inference that it is possible to measure an individual's probability of remembering an image. To measure the probability that a person will remember an image, the person is presented with a stream of images. This process is called Visual Memorability Game (Isola et al., 2011a). The stream of images contained two kinds of images, targets, and fillers. The annotator is shown images one by one, where the image is displayed for 2.4 seconds. Between each target image multiple filler images are shown unbeknownst to the annotator. On a random manner, previously shown target images are repeatedly shown now and then. When each image is being shown, the annotator is asked to press a key in the keyboard if that annotator feels that the target image is being repeated in the stream. Based on this, then the percentage of times the annotator has correctly identified repeated target images will be checked and is annotated as the memorability of the target image from one annotator. The same set of images are shown to multiple more people in the same manner. So, the approximate memorability scores will be obtained for the same image from multiple people. Image memorability is a reflection of individual viewing the image, but however, the level of memorability of an image is quite similar across individuals most of the time (Sommer et al., 2021). So, since the memorability of the image is only going to slightly vary for most people, these approximate measures are taken as the ground truth memorability score. The slight difference between most deep learning datasets and image memorability datasets is that, for each image, we'll have multiple annotations, that is, multiple memorability scores, which is fine because most of them aren't going to vary that much.

As mentioned earlier, unlike more objective properties of images such as photo composition or image quality, image memorability cannot be objectively defined and hence might slightly vary from person to person. However, generally, humans agree with each other on certain common

Peer) Comput. Sci. reviewing PDF | (CS-2021:05:61139:2:0:NEW 23 Sep 2021) 
122 factors that tend to make an image more memorable despite this large variability. Factors like 123 color harmony and object interestingness are generally agreed upon by people as factors that 124 improve image memorability (Khosla et al., 2015a).

125 Few methods have been proposed (Perera, Tal, \& Zelnik, 2019; Fajtl et al., 2018; Squalli126 Houssaini et al., 2018) to predict the memorability of an image using deep learning methods. 127 Those methods either used handcrafted features or ensemble models to predict the memorability 128 score. Ensemble models are hard to train, computationally expensive and are prone to overfitting 129 (Canchumuni, Alexandre \& Pacheco, 2019). The overfit models normally do not perform well 130 on different kinds of images that are not in the training set, while computationally expensive 131 models are not suitable for deployment to real world on web servers or computers with low memory GPUs and real-world deep learning systems are heavily reliant on computers with GPUs. Methods that use handcrafted features along with machine learning models are not accurate on different kinds of images because is it extremely hard to handcraft a comprehensive amount of features that can span a wide distribution. The idea of using data-driven strategies to predict image memorability was first introduced by (Isola et al., 2011a). The Visual Memorability Game was used to prepare the images in the Isola et al. dataset and annotate their respective memorability score. The game was run on Amazon Mechanical Turk, where users were presented with a stream of images with some images repeating on a random basis. The users were asked to press a key when they believe that the image displayed was already seen before. In the Isola et al. dataset, they have collected 2222 images along with the annotated memorability scores. Since memorability can vary slightly from person to person, each image was shown to 78 participants on an average, when the annotators played the Visual Memorability Game. Each image being tagged more than once accounts for the slight variation in memorability among people. This also means that when the deep learning model is being trained, during each epoch, the model will be given the same image as input multiple times but with a slightly varying ground truth. When they analyzed the images and their memorability scores together, they have understood that the memorability of an image is highly related to a certain object and scene semantics such as 'Labelled Object Counts,' 'Labelled Object Areas' and 'Object Label Presences.' Also, when each image was segregated into scene categories, it was inferred that much of what contributes to the image's memorability score was from both the object and scene semantics. They have followed up on their work to understand the human-understandable visual attributes to understand memorability as a cognitive process. They have developed a deep learning model that can predict scene category of an image to with another deep learning model that predicts image memorability to understand and identify a compact set of image properties that affect image memorability (Lu et al., 2020). A new dataset, Large-scale Image Memorability dataset (LaMem) which is publicly available, is a novel and diverse dataset with 60,000 images, each tagged with memorability score similar to the dataset by Isola et al. The authors (Khosla et al., 2015a) have used Convolutional Neural Networks (MemNet) to fine-tune deep features that outperform all other features by a large margin. The analysis made by the author on the responses of high-level Convolutional Neural 
162 Networks (CNN) layers shows which objects are positive. A new computational model based on

163

164

165

166

167

168

169

170

171

172

173

174

175

176

177

178

179

180

181

182

183

184

185

186

187

188

189

190

191

192

193

194

195

196

197

198

199

200

201

an attention mechanism to predict image memorability based on deep learning was proposed. In this paper, the authors have shown that emotional bias affects the performance of the proposed algorithm due to the deep learning framework arousing negative pictures than positive or neutral pictures (Baveye et al., 2016).

Squalli-Houssaini et al. presented a hybrid CNN with Support Vector Regression (SVR) model trained on the LaMem dataset. The model achieved an average rank correlation of 0.64 across the validation sets. Based on the predictions, the correlation between interestingness and memorability was analyzed. The predictions were compared using the Flickr Interestingness API and the results showed that memorability did not correlate much with interestingness (SqualliHoussaini et al., 2018).

Visual attention has a huge effect on image memorability (Fajtl, J et al., 2018) . However, very little work has been done on taking advantage of visual attention to predict image memorability. Mancas and Meur proposed a model that uses a new set of attention-driven features by identifying the link between image saliency and image memorability. The model achieved a $2 \%$ increase in performance from the existing models. It was also inferred that images with highly localized regions are more memorable than those with specific regions of interest (Mancas M \& Le Meur, 2013).

A novel deep learning architecture was proposed that took advantage of the visual attention mechanism to predict image memorability by (Fajtl, J et al., 2018). The architecture made use of a hybrid of Feedforward CNN architecture and attention mechanism to build a model that can help build attention maps and, in turn, predict memorability scores. The model attained excellent results, but the biggest downside was overfitting and lack of the provision to use transfer learning swiftly. The model also contains a large number of parameters making it hard for real-time production.

Another model that used visual attention mechanism was proposed by (Zhu et al., 2020). The architecture is a multi-task learning network that was trained on LaMem dataset and AADB dataset (Kong et al., 2016) to predict both the memorability score and aesthetic score of an image, hence it was also trained using two datasets at the same time, one for image memorability and the other for image aesthetics. The model used a pixelwise contextual attention mechanism to generate feature maps. Even though this model was able to use transfer learning, the attention mechanism used is computationally expensive, especially if the number of channels in the intermediate layers is high. This model for the memorability task achieved a rank correlation of only 0.660 , which is a much lower score than the ones achieved other existing models.

An ensemble model that predicts video memorability was proposed by (Zhao $T$ et al., 2021). The model was trained on the MediaEval2020 dataset and is an ensemble of models that extract audio, video, image and text features from the input to predict video memorability. The features of the audio were extracted using a pretrained VGG model, while the image and video features were extracted using a ResNet-152. These features were then passed onto other machine learning models to get the memorability score. It was found that Bayesian Ridge Regressor worked best

Peer) Comput. Sci. reviewing PDF | (CS-2021:05:61139:2:0:NEW 23 Sep 2021) 
202 for processing audio features while a Support Vector Regressor worked best for processing 203 image and video features. The text features for the tagged human annotated captions were 204 obtained using GloVe word embeddings. The model achieved a rank correlation of 0.370 for 205 short term memorability and 0.289 for long term memorability on the validation set of the 206 dataset.

207 A multi-modal fusion-based model trained on the MediaEval2019 dataset for video memorability 208 prediction was proposed by (Leyva R and Sanchez V, 2021). This model takes advantage of 209 motion estimation techniques and combines it with text, audio and image features. To estimate 210 motion and obtain its feature vectors, two 3DResNets were used. The image features were 211 extracted using ResNet-56 and ResNet-152, while the text features were obtained using a 212 combination of CNN and Gated Recurrent Unit (GRU). The feature vectors from text, image, 213 and motion estimation are then processed through late fusion and then a Bayesian Ridge 214 Regressor predicts the memorability score. On the validation set, the model obtained a rank 215 correlation of 0.5577 for short term memorability and 0.3443 for long term memorability.

216 A Hidden Markov Model (HMM) produced using Variational Hierarchical Expectation 217 Maximization was proposed by (Ellahi et al., 2020). A new dataset with 625 images was tagged 218 by 49 subjects. During the data annotation session, an eye-gaze camera setup was used to track 219 the eye-gaze of each subject when they were presented with a stream of images. The goal of this 220 setup was to analyze how much eye gaze contributed to image memorability. The model 221 achieved an accuracy of only $61.48 \%$ when the ground truth eye gaze and predicted eye gaze 222 were compared.

223 A novel multiple instance-based deep CNN for image memorability prediction was proposed that 224 shows the performance levels that are close to human performance on the LaMem dataset. The 225 model shows EMNet, automatically learns various object semantics and visual emotions using 226 multiple instance learning frameworks to properly understand the emotional cues that contribute 227 extensively to the memorability score of an image (Basavaraju, \& Sur, 2019).

228 The main problem with the previously proposed state of the art models is that they are 229 computationally intensive. Some of the previously proposed models are not suitable for 230 production purposes. Most of the previously proposed models constitute several pre-processing 231 stages and use multiple CNNs in a parallel manner to provide results. The issues that accompany 232 these strategies are over-fitting, high computational complexity and high memory requirements. 233 To solve these issues, there is a need for an approach that results in a smaller number of 234 parameters and a model that contains layers that can prevent overfitting.

235 Therefore, to solve the above-mentioned issues, in this work, the proposed Residual Memory Net 236 (ResMem-Net) is a novel deep learning architecture that contains fewer parameters than previous 237 models, making it computationally less expensive and hence is also faster during both training 238 and inference. ResMem-Net also uses 1x1 convolution layers and Global Average Pooling 239 (GAP) layers, which also helps to reduce the chances of overfitting. In this model, a hybrid of 240 Convolutional Neural Networks and Long Short-Term Memory Networks (LSTM) is used to 241 build a deep neural network architecture that uses a memory-driven technique to predict the 
242 memorability of images. ResMem-Net achieves results that are very close to human performance

243 on the LaMem dataset. Transfer learning is also taken advantage of during the training process, 244 which has helped ResMem-Net to generalize better.

245 The publicly available LaMem dataset is used to train the model, consisting of 60,000 images, 246 with each image being labeled with a memorability score. The proposed architecture has given 247 close to human performance with a rank correlation of 0.679 on the LaMem dataset. Finally, 248 heatmaps have been generated using Gradient Regression Activation Map (GradRAM) technique 249 (Selvaraju et al., 2017), which is used the visualize and analyze the portions of the image that 250 causes the image to be memorable. Even though this paper focuses on the results of the LaMem 251 and Isola et al. dataset, the key contribution of this paper is the novel ResMem-Net Neural 252 Network architecture which can be used for any other classification or regression task in which 253 the intermediate features of the $\mathrm{CNN}$ might be useful.

254

255

256

257

258

259

260

261

262

263

264

265

266

267

268

269

270

271

272

273

274

275

276

277

278

279

280

281

In the materials \& methods section, the proposed architecture, the novelty of the architecture, loss function, and the datasets used are explained in detail. The use of transfer learning, optimization function, evaluation metrics, loss function and weight update rule are also discussed in the materials \& methods sections. In the experiments and results section, the experimental setup, hyperparameters used, training settings the results of the model are discussed. The results are compared in detail with existing works and a qualitative analysis done to understand memorability is also discussed. Finally, in the conclusions section, the proposed work and results are summarized and then the potential future enhancements are discussed..

\section{Materials \& Methods}

This section deals with the proposed Neural Network architecture, the dataset used, and the evaluation of the proposed model's performance. Further, the results obtained from an extensive set of experiments are compared with previous state-of-the-art results. It shows the superiority of the proposed architecture; for every problem solved by deep learning, four core entities have to be defined before the results are obtained. They are the dataset, the neural network architecture, the loss function and the training procedure.

\section{Deep Hybrid CNN for the prediction of memorability scores}

This section provides a detailed explanation of the ResMem-Net. A visual depiction is given in Figure 1. The figure shows that there are two distinct portions in the entire architecture. At the top of ResMem-Net, ResNet-50 (He et al., 2015) is used as the backbone, state of the art deep learning architecture for many applications. ResNet-50 is a 50-layer deep neural network that contains convolution kernels at each layer. The main innovation in ResNet-50 is the skip connection which helps to avoid vanishing gradients in very deep neural networks. The skip 
282 connection is present at every convolutional kernel present in the ResNet-50 model. The skip 283 connection adds the input of the convolutional kernel to the output, hence allowing the model to 284 propagate information to the next layer even if the output of the convolutional kernel is too small 285 in terms of numerical value. This is how ResNet-50 and other variants of ResNet are not prone to 286 vanishing gradient problem (He et al., 2015). Since there are going to be 50 convolutional 287 kernels in ResNet-50, it is a Deep CNN. The input image is given to ResNet-50, and the size of 288 the image used in our experiment is $224 \times 224$ px. One of the core features of the proposed 289 architecture is that the CNN part of the architecture is fully convolutional, and due to the use of 290 Adaptive Average Polling layers, the model isn't constrained to the size of the input image hence 291 the input image can be higher or lesser than $224 \times 224$ px size.

292

293

At the bottom of ResMem-Net, a Long Short-Term Memory (LSTM) unit is responsible for 294 predicting the output, the memorability score. LSTM is an enhancement to Recurrent Neural Networks (RNN). RNNs are generally used for sequential data such as text-based data or time-

296

297 series data. However, in RNN, there are no memory units to resolve any long-term dependencies (Cho et al., 2014). Several variants of LSTM were analyzed, and it showed that the standard LSTM model with forget gate gave the best results on a wide variety of tasks (Greff et al., 2019).

299

300

301

302

303

304

305

306

307

308

309

310

311

312

313

314

315

316

317

318

319

320

321 In an LSTM unit, a 'cell state' is computed that can retain information from previous input sequences. The cell state is computed using 'forget gate' and 'output gate' as demonstrated in Figure 2. These gates determine which information from previous layers should be removed from the cell state vector and which information should be retained. LSTM units accept sequential data as inputs, and in this architecture, the input to the LSTM unit are the activations of the hidden layers of the ResNet-50 model, as shown in Figure 2. As the input sequences being sent to the LSTM unit must be of the same size, Global Average Pooling (GAP) is used to shrink the activations of the hidden layers to a size of $(\mathrm{Cx} 1 \mathrm{x} 1)$ where $\mathrm{C}$ is the number of channels. Global Average Pooling is very much like Densely connected Layers in Neural Networks because it performs a linear transformation on a set of feature maps. This allows us to ensure that there is no need to care too much about the size of the output activations at each layer and the input image's size. As studied in (Hsiao et al., 2019), Global Average Pooling also does not have any parameter to optimize, thus avoiding overfitting and reducing computational needs. GAP layers can be thought of as an entity, that enforces the feature maps (outputs of intermediate layers) to be the confidence maps of various intrinsic features of the input image. Hence, GAP also acts as structural regularizers without requiring any hyper parameters. Also, global average pooling sums the spatial information, hence they are also robust to any spatial changes in the feature maps. Further, a convolution operation is done on the output of GAP layers to obtain a 128-channel output which can be flattened to obtain a vector of Rank 128.

The main reason behind passing the hidden layer activations to the LSTM unit is to ensure that the cell state vector can remember and retain the important information from the previous hidden layers. When the final layer's activation is passed to the LSTM unit, the important information of the previous layers along with the final layer's activation is obtained and then all that 
322 information is used to compute the memorability score. The LSTM layer's output is an n323 dimensional vector, passed to a linear fully connected layer that gives a scalar output, which is 324 the memorability score of the image. This strategy allows us not just to use the final layer's 325 activations alone which is generally done in previous works discussed.

326

327

328

329

330

331

332

333

334

335

336

337

338

339

340

341

342

343

344

345

346

347

348

349

350

351

352

353

354

355

356

357

358

359

\section{Mathematical Formulation of the model}

So, the input image is a tensor of size $(3,224,224)$, denoted by $A_{0}$. The output of $L^{\text {th }}$ identity block is denoted by $A_{L}$, as shown in equations (1) and (2). At each $L^{\text {th }}$ identity block, the output of the identity block is calculated by:

$$
Z_{L}=W_{L} \otimes A_{L-1}
$$

$A_{L}=\operatorname{relu}\left(Z_{L}\right)$ where $\operatorname{relu}(a)=\max (0, a)$

where,

$\mathrm{Z}_{\mathrm{L}}$ is the output of the $\mathrm{L}^{\text {th }}$ identity block,

$A_{L}$ is the output of the activation function with $Z_{L}$ as input.

For all $\mathrm{L}, \mathrm{A}_{\mathrm{L}}$ is passed through a Global Activation Pooling layer, which converts a $(\mathrm{C}, \mathrm{W}, \mathrm{H})$ tensor to a $(C, 1,1)$ tensor by taking the average of each channel in the activation matrix $A_{L}$.

At the LSTM layer, the initial cell state is denoted by $\mathrm{C}_{0}$, and $\mathrm{h} 0$ denotes the initial activation. Before the hidden layer activations are passed to the LSTM, $\mathrm{C}_{0}$ and $\mathrm{h}_{0}$ are initialized as random vectors using 'He' initialization strategy to help avoid the exploding gradient problem (He at al., 2015).

The LSTM unit consists of three important gates that form the crux of the model:

1. Update Gate - Decides what information should be remembered and what information should be thrown away

2. Forget Gate - To decide which information is worth storing

3. Output Gate - The output of the LSTM unit

Update Gate : $\mathrm{G}_{\mathrm{u}}=\operatorname{sigmoid}\left(\mathrm{W}_{\mathrm{hc}} \mathrm{c}^{\langle t-1\rangle}+\mathrm{W}_{\mathrm{ub}} \mathrm{x}^{\langle t\rangle}+\mathrm{b}_{\mathrm{u}}\right)$

Forget Gate : $\mathrm{G}_{\mathrm{u}}=\operatorname{sigmoid}\left(\mathrm{W}_{\mathrm{fc}} \mathrm{c}^{\langle t-1\rangle}+\mathrm{W}_{\mathrm{fx}} \mathrm{x}^{\langle t\rangle}+\mathrm{b}_{\mathrm{f}}\right)$

Output Gate : $\mathrm{G}_{\mathrm{o}}=\operatorname{sigmoid}\left(\mathrm{W}_{\mathrm{oc}} \mathrm{C}^{\langle t-1\rangle}+\mathrm{W}_{\mathrm{ox}} \mathrm{x}^{\langle t\rangle}+\mathrm{b}_{\mathrm{u}}\right)$

Hidden cell state : $\quad \mathrm{h}^{\langle\downarrow\rangle}=\mathrm{G}_{\mathrm{u}}+\mathrm{h}^{\langle t\rangle}+\mathrm{G}_{\mathrm{f}} * \mathrm{~h}^{\langle t-1\rangle}$

LSTM output : $\mathrm{C}^{\langle t\rangle}=\mathrm{G}_{\mathrm{o}} * \mathrm{~h}^{\langle\mathrm{t}\rangle}$

The output of $\mathrm{G}_{\mathrm{u}}, \mathrm{G}_{\mathrm{f}}, \mathrm{G}_{\mathrm{o}}, \mathrm{h}^{<\downarrow}$, and $\mathrm{c}^{<\downarrow}$ can be calculated using the formulas given in equations (3), (4), (5), (6) and (7), respectively.

\section{The Loss Function}

The scores of the images in both the mentioned datasets are continuous-valued outputs, making this entire task a regression task. To understand how good our model predicts memorability, loss functions are used, which can approximate the divergence between the target distribution and the predicted distribution. Generally, for regression tasks, the L2 loss function, also known as the 
360 361

362

363

364

365

366

367

368

369

370

371

372

373

374

375

376

377

378

379

380

381

382

383

384

385

386

387

388

389

390

391

392

393

394

395

396

397

398

Mean Squared Error (MSE), is used as the loss function for the proposed model and the formula is given in equation (8).

$$
\operatorname{MSE}=\frac{1}{\mathrm{n}} \sum_{\mathrm{i}=1}^{\mathrm{n}}\left(\mathrm{y}_{\mathrm{i}}-9 \%\right)^{2}+\lambda \Sigma(\theta)^{2}
$$

Where the $(\tilde{y})$ represents the predicted value, while $y_{\mathrm{i}}$ represents the ground-truth value of the $\mathrm{i}^{\text {th }}$ image in the dataset, $\lambda$ represents weight decay and $\theta$ represents the weights. The second term is added to the existing loss function to prevent the model from overfitting. The regularization procedure is known as L2 regularization, which multiplies a weight decay (hyperparameter) and the summation of all the weights used in the Neural Network. The weight decay prevents the weights from being too big, which ultimately prevents the model from overfitting.

\section{Pseudocode for ResMem-Net}

The pseudocode for the forward-pass of ResMem-Net is given below. Initially, the information passed through each layer in the backbone by passing the previous layer's output to the next layer. Each time an output from a layer is obtained, the outputs are passed to a global average pooling layer, which works as depicted in the function called globalAveragePooling. The outputs from the globalAveragePooling method are passed to the LSTM_CELL at each iteration. After the final iteration, the memorability score can be retrieved from the LSTM_CELL.

Procedure mem (images):

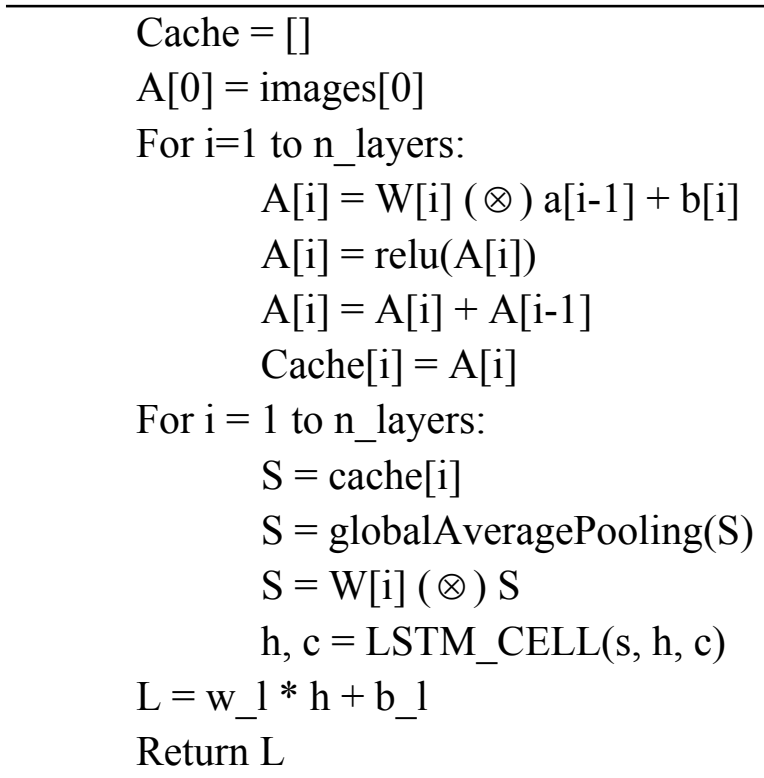

Procedure LSTM_CELL $\left(\mathrm{x}, \mathrm{h}_{\mathrm{t}-1}, \mathrm{c}_{\mathrm{t}-1}\right)$ : 
399

400

401

402

403

404

405

406

407

408

409

410

411

412

413

414

415

416

417

418

419

420

421

422

423

424

425

426

427

428

429

430

431

432

433

434

435

436

437

438

$$
\begin{aligned}
& \text { ot }=\operatorname{sigmoid}\left(\mathrm{W}_{\mathrm{xo}} * \mathrm{x}+\mathrm{W}_{\mathrm{ho}} * \mathrm{~h}_{\mathrm{t}-1}+\mathrm{W}_{\mathrm{co}} * \mathrm{ct}+\mathrm{b}_{\mathrm{o}}\right) \\
& \mathrm{ht}=\mathrm{ot} * \tanh (\mathrm{ct}) \\
& \text { return ht, ct }
\end{aligned}
$$

Procedure globalAveragePooling(tensor):

$$
\begin{aligned}
& \mathrm{c}, \mathrm{h}, \mathrm{w}=\operatorname{dimensions}(\text { tensor }) \\
& \text { for } \mathrm{i} \text { in } \operatorname{range}(\mathrm{c}): \\
& \qquad \begin{array}{l}
\operatorname{Avg}=(1 / \mathrm{h}) *(\text { tensor}[\mathrm{i}]) \\
\text { tensor }[\mathrm{i}]=\operatorname{Avg}
\end{array}
\end{aligned}
$$

return tensor

In figure 3, the pipeline used during this research is depicted. The process starts with data collection and processing and then proceeds with the model development phase. In the model development phase, the model's architecture is initially defined, modified to our task and finally programmed. Then the training phase is done with the given datasets, and finally, hyperparameter tuning is done, where various batch sizes, learning rates and residual models are tried to find the optimal settings. Then to analyze the results, GradRAM technique is used to visualize the activation maps to understand how the model predicts the results.

\section{Dataset Used}

In this paper, two publicly available datasets are used: the LaMem dataset and dataset from Isola et al. LaMem is currently the largest publicly image memorability dataset that contains 60,000 annotated images. Images were taken from MIR Flickr, AVA dataset, Affective images dataset, MIT 1003 dataset, SUN dataset, image popularity dataset and Pascal dataset. The dataset is very diverse as it includes both object-centric and scene-centric images that capture a wide variety of emotions. The dataset from Isola et al. contains 2222 images from the SUN dataset. Both datasets were annotated using the Visual Memorability Game. Amazon Mechanical Turk was used to allow users to view the images and play the game which helped annotate the images.

Both the datasets were collected with human consistency in mind, i.e., the authors ran human consistency tests to understand how consistent the users are able to detect repetition of images. The consistency was measured using Spearman's rank correlation, and the rank correlation for LaMem and Isola et al. are 0.68 and 0.75 respectively. The human consistency was calculated by inviting a new set of participants to play the Visual Memorability Game. The participants were split into two halves and were asked to independently play the game for the images in the datasets. Then, the human consistency was measured by how similar the second half the participants' memorability scores were to the memorability scores obtained from the first half of the participants. This analysis show that humans are generally consistent when it comes to remembering or forgetting images. Also, for both the datasets, the authors of the datasets have themselves provided the dataset splits along with the dataset. Those files contain both the ground truth values of each image and information about whether they belong to the training or 
439 validation sets. In the LaMem dataset, 45,000 images are given for training, while 10,000 images

440 for validation.

441

442 Optimization

443 The loss function is actually differentiable and is also a function of the parameters of the Neural

444 Network. The gradient of the loss function concerning the weights can guide us through a path to

445 allow us to identify the right set of parameters that yield a low loss using gradient descent-based

446 methods. In our experiments, a slightly modified method of ADAM optimizer is used, which is a

447 combination of Stochastic Gradient Descent with Momentum and RMSprop added with the cost

448 function (Yi, Ahn, \& Ji, 2020) The loss function of Neural Networks is very uneven and sloppy

449 due to the presence of too many local minima and saddle points. This modified version of

450 ADAM uses exponentially weighted moving averages. Initially, compute the momentum values

451 are computed using equations (9), (10) and (11):

452

453

454

455

456

457

458

459

460

461

462

463

464

465

466

467

468

469

After the momentum values are calculated, the update rule for the weights is done using equation 12 ,

$$
\mathrm{H}(\theta)=\lambda \mathrm{J}(\theta)+\frac{\partial \mathrm{J}(\theta)}{\partial \theta}
$$

$$
\text { where, } \mathrm{J} \text { - cost function }
$$

$\lambda$ - scaling constant (hyperparameter)

$\theta$ - weights

$$
\mathrm{m}_{\mathrm{i}}=\alpha \mathrm{m}_{\mathrm{i}-1}+(1-\alpha) \mathrm{H}\left(\theta_{\mathrm{i}}\right)
$$

$$
\mathrm{v}_{\mathrm{i}}=\beta \mathrm{v}_{\mathrm{i}-1}+(1-\beta)(\mathrm{H}(\theta \mathrm{i}))^{2}
$$

where, $\alpha, \beta$ - scaling constant

$$
\mathrm{m}_{\mathrm{i}}, \mathrm{v}_{\mathrm{i}}-\text { first, second momentum }
$$

$\mathrm{m}_{0}, \mathrm{v}_{0}$ - initial momentum (set to 0 )

470

471

472

473

$$
\theta_{i+1}=\theta_{i}-\eta \frac{\hat{\mathrm{m}}_{\mathrm{i}}}{\sqrt{\hat{\mathrm{v}}_{\mathrm{i}}+\varepsilon}}
$$

$$
\text { Where, } \theta_{i} \text { - current weight }
$$

$$
\theta_{\mathrm{i}+1} \text { - updated weight }
$$

$$
\eta \text { - learning rate }
$$

$$
\hat{\mathrm{m}}_{\mathrm{i}}=\frac{\mathrm{m}_{\mathrm{i}}}{(1=\alpha)} ; \hat{\mathrm{v}}_{\mathrm{i}}=\frac{\mathrm{v}_{\mathrm{i}}}{(1-\beta)}
$$

$$
\varepsilon \text { - constant to avoid zero division (usually } 10^{-6} \text { ) }
$$


475

476

477

478

479

480

481

482

483

484

485

486

487

488

489

490

491

492

493

494

495

496

497

498

499

500

501

502

503

504

505

506

507

508

509

510

511

512

513
Adding the cost function to the gradient of the weights w.r.t the cost functions ensures that the loss landscape is much smoother and can converge at a good minimum. This helps because, even if the gradient of the cost function w.r.t weights are very small, adding the scaled version of the cost function ensures that the weights keep changing, ensuring that the model doesn't get stuck in local minima or saddle points.

\section{Learning Rate and One Cycle Learning Policy}

The learning rate is one of the most important hyperparameters in deep learning as it decides how quickly the loss moves towards a minimum in the loss function's surface. Learning rates can decide whether a model converges or diverges over time. If a high learning rate is used throughout the training process, loss of the model may diverge over some time, but if it is set to a low value, then the model may take too much time to converge. To solve this issue, generally the learning rate is reduced over time using a decaying function. But decaying functions can lead to the model's parameters to be stuck in saddle points or in local minima, which can lead to the model not learning new parameters in the consecutive epochs. To avoid these issues, (Smith, 2018) has proposed a method called One Cycle Learning. In one cycle learning policy, for each epoch, the learning rate is varied between a lower bound and upper bound. The lower bound's value is usually set at $1 / 5$ th or $1 / 10$ th of the upper bound.

In one cycle learning, each epoch is split into 2 steps of equal length. All deep learning models are trained using mini-batches, so if the dataset has 100 batches, the first 50 batches are included in step 1 and the rest are included in step 2. During the start of each epoch, the learning rate is set to the lower bound's value and at the end of each mini-batch, the learning rate is slowly increased to ensure that the learning rate reaches the upper bound by the end of step 1 . In step 2, the training proceeds with the upper bound as the learning rate and then the learning rate is slowly decayed after each mini-batch, to ensure that by the end of step 2, the learning rate is back to lower bound. This is then repeated for each epoch. Varying the learning rate between a high and a low value allows the model to escape the local minima or saddle points. The higher learning rate allows the model to escape local minima and saddle points during training, while the lower learning rate ensures that the training leads to parameters that ensure a lower loss in the loss function.

\section{Transfer learning}

Transfer learning is training a model on a large dataset and retraining the same model on a different dataset with lesser data. Intuitively, the learned features from larger datasets are used to help improve accuracy on datasets with smaller data points. In our work, a pre-trained ResNet-50 that is trained on the ImageNet dataset is used, which contains 3.2 million images, with each image categorized in one among the 1000 categories.

Peer] Comput. Sci. reviewing PDF | (CS-2021:05:61139:2:0:NEW 23 Sep 2021) 
514 In our work, the semantic features learned through ImageNet will allow the model to be quickly 515 trained and perform better on identifying the memorability of images in the validation dataset.

516 The feature maps in the pretrained ResNet-50 will contain feature maps for objects, scenes and 517 other visual cues that aren't present in the images present in the datasets that are used to train the 518 model. This is so because, the pretrained ResNet-50 was trained on a dataset with diverse set of 519 images. So, after careful retraining, many of these feature maps in the pretrained model will be 520 retained. This will allow the re-trained model to identify the objects and scenes not present in the 521 LaMem and Isola et al. datasets, which can drastically improve real-time deployment 522 performance. Empirical evidence for the above explanation is given in (Rusu et al, 2016).

523

524

525

526

527

528

529

530

531

532

533

534

535

536

537

538

\section{Experiments and Results}

In this section, the evaluation criteria, and outcome of the experiments are discussed. The training settings i.e, the hyperparameters, hardware used, training and validation splits of the dataset are discussed. Finally, the outcome of the training process and the reason behind superior results are explained.

\section{Evaluation Metric}

The L2 loss function is generally a good metric to find how well the proposed model performs, but here the Rank Correlation method is also used to evaluate the proposed model. The Spearman Rank Correlation $(\rho)$ is computed between the predicted score and target score, is used to find the consistency between the predicted scores and target score from the dataset. The value of $\rho$ ranges from -1 to 1 . If the rank correlation is extremely close to 1 or -1 , then it means that there is a strong positive or negative agreement respectively between the predicted value and ground truth, while a rank correlation of 0 represents that there is complete disagreement. The rank correlation between predicted and target memorability score is given by the equation (9):

$$
\rho=1-\frac{6 \cdot \sum_{\mathrm{i}=1}^{\mathrm{n}}\left(\mathrm{r}_{\mathrm{i}}-\mathrm{S}_{\mathrm{i}}\right)^{2}}{\mathrm{n}^{3}-\mathrm{n}}
$$

539

540

Where $r_{i}$ is the ground truth while $s_{i}$ is the predicted value from the model, whereas $n$ is the

541 number of images in the dataset.

\section{Training Settings and Results}

543 The batch size was set at 24 throughout the training process and the images were resized to a size 544 of $224 \times 224$. Since transfer learning is employed, when the backbone's (ResNet-50) parameters 545 were freezed, the upper bound and lower bound for the learning rate was set at 0.01 and 0.001 546 respectively. After 10 epochs, the backbone's parameters were unfreezed and then the upper 547 bound and lower bound for the learning rate was set at 0.001 and 0.0001 for 15 epochs. For the 548 rest of the epochs, the lower bound and upper bound for the learning rate were set at 0.0001 and 5490.00001 respectively. The training process consisted of a total of 40 epochs. For regularization, 550 a value of 0.0001 was set as L2 weight decay. The model was trained on a Nvidia Quadro P5000 551 GPU which has 16GB GPU memory and 2560 CUDA cores. 
552 To ensure stable training, normalization and dropout layers were used. The authors of the 553 LaMem dataset have given 5 training set splits because each image has multiple annotations. 554 Hence, 5 different models were trained, one for each split, and the results were averaged across 555 the models while testing. For cross-validation purposes, the authors of the LaMem datasets 556 divided the dataset into five sets, where each set contains 45,000 images for training, 10,000 557 images for testing and 3,741 images for validation purposes. After the training the model using 558 the above settings, ResMem-Net obtained an average rank correlation of 0.679 on the LaMem 559 dataset and 0.673 on the Isola et al. dataset as mentioned in table 1 and table 2. These results 560 indicate that the use of a hybrid of pretrained CNN and LSTM has contributed to the increase in

561 the accuracy of the model. Also, the model contains only a ResNet-50 backbone and a LSTM 562

563

564

565

566 unit, making it computationally less expensive.

567

568

569

570

571

572

573

574

575

576

577

578

579

580

581

582

583

584

585

586

587

588

589

590

591

\section{Discussion}

In this section, the results of the experimental outcomes are taken and compared with existing models on two datasets, namely, LaMem and the Isola et al. dataset. Then, the comparison the number of parameters present in the existing models and the proposed model is done to establish why the proposed model has lesser memory requirements and to establish why the model is better suited for deployments to servers or other production needs. Finally, the results of the qualitative analysis done using the GradRAM method is presented to understand which regions of the image lead to higher memorability scores and to answer the question, "What makes an image more memorable?".

The Spearman Rank Correlation metric has been used to evaluate the models and consistency of the results. Since each image has been annotated by multiple subjects, the rank correlation metric is better suited than the L2 loss to compare how consistently the models are predicting memorability scores. The five models discussed in the introduction are considered and the average of results is compared with the previous works. To ensure that the comparison of our results is fair, as mentioned in results sections, five models were trained on the five sets and found the average of the results. The models used for comparison were also trained in the same way by the respective authors, hence that ensures that the differences between the results of the previous models is due to the models only, not due to any other reasons.

Table 1 represents the results of various models on the LaMem dataset and table 2 represents results of various models on the Isola et al. dataset. Table 1 and figure 4 show that ResMem-Net attains a rank correlation of 0.679 , a $6.09 \%$ increase from MemNet, a 35\% increase from CNNMTLES, a $2 \%$ increase from MCDRNet and a 1.2\% increase from EMNet. The human-level accuracy on LaMem is 0.68 , and ResMem-Net has brought us extremely close to human accuracy with a difference in rank correlation of just 0.001 .

From table 2 and figure 5, it can be inferred that ResMem-Net attains a rank correlation of 0.673 , which is a $10.33 \%$ higher from MemNet, 5.48\% increase from MCDRNet, $1.4 \%$ increase from 
592 EMNet and a $45.67 \%$ increase from SVR. The authors have not provided the human accuracy for 593 this dataset. Hence, it is not possible to tell how close ResMem-Net is to human accuracy for the 594 Isola et al. dataset, but it is clear that ResMem-Net has outperformed all other previous works. 595 The reason behind the superior performance can be attributed to the use of LSTM unit, modified 596 optimization function, pretrained ResNet-50 backbone and the use of cyclic learning rates.

597 Tables 3 and 4 depict the predicted scores on various sets of images on the dataset. In both the 598 tables, the images are arranged in descending order of the predicted memorability scores. For 599 example, the 'Top 10' row depicts the average of the top 10 highest predicted memorability 600 scores that are predicted by various network architectures and finally, the average of the ground 601 truth of the same images is also given in the same row. The results are based on average over the 5 -fold cross-validation tests as provided by the creators of the datasets.

608

609

From both Tables 3 and 4, it can be inferred that, on average, ResMem-Net performs better than previously proposed models on both Isola et al. dataset and LaMem dataset. For the top 10, EMNet predicts a memorability of $91.89 \%$ and $82.43 \%$ on the LaMem and Isola et al. datasets, respectively, while ResMem-Net predicts an average memorability of $93.82 \%$ and $82.61 \%$ on the same LaMem and Isola et al. datasets, respectively. MCDR-Net obtained an average memorability $93.15 \%$ and $81.75 \%$, while MemNet has obtained $91.7 \%$ and $80.16 \%$ on the LaMem and Isola et al. datasets respectively for the top 10. When compared to the ground truth, which is $100 \%$, these scores clearly state that ResMem-Net is more consistent with the images with high memorability. On the other hand, when for the 'Bottom 10' images, EMNet predicts an average memorability of $48.41 \%$ and $27.42 \%$, while for MCDRNet it is $50.94 \%$ and $26.52 \%$ on the LaMem and Isola et al. datasets, respectively. In comparison, ResMem-Net predicts an average memorability of $47.9 \%$ and $27.42 \%$, respectively. Again, when comparing those results to the ground truth values, which is $33.57 \%$ and $5.69 \%$ for LaMem and Isola et al. datasets respectively, ResMem-Net provides similar results to EMNet.

619 It would also be unfair to completely ignore the results of traditional machine learning algorithms on image memorability. Despite many empirical results that depict the superiority of deep learning algorithms on computer vision tasks, certain studies have shown that the use of hand-crafted features when ensembled with machine learning algorithms such as SVR or Random Forests can, in fact, provide better results. Of course, concerns regarding the generalization of the models on new data have been raised, which are the very papers that propose the non-deep learning-based strategies themselves. However, in table 5 and figure 6 , it is very clear that the proposed ResMem-Net quite easily outperforms traditional machine learning strategies.

628 Since the results are from the validation set, it is clear that the model did not overfit but rather learned features that contribute to the memorability scores of the image. The validation set also encompasses a wide variety of landscapes and events, which also leads us to believe that the 631 model performs well on different kinds of images. 


\section{Computational Complexity Analysis}

633 This section deals with the comparison of the computational complexity of various previous

634 models and ResMem-Net. It has been already established that ResMem-Net is quite minimal 635 compared to other previously proposed models in network parameter size. Table 6 shows the 636 number of parameters or weights present in ResMem-Net, MemNet, MCDRNet and EMNet. It is 637 clear from the table that ResMem-Net has a significantly much lesser number of parameters than 638 the previously proposed network architectures. CNN's are composed of convolution operations, 639 which are very much compute intensive. So, the lesser the number of parameters, the faster the 640 model takes to provide outputs. The bigger advantage of ResMem-Net is that it has a 641 significantly lesser number of weights and still provides better accuracy on both LaMem and 642 Isola et al. datasets. The time taken for ResMem-Net to process an image of size $512 \times 512 \mathrm{px}$ is 643 approximately $0.024 \mathrm{~s}$ on Nvidia Quadro P5000 GPU. It should also be noted that having too 644 many parameters can cause overfitting and hence ResMem-Net is less prone to overfitting 645 because it has a significantly lower number of weight parameters.

646

647 Qualitative analysis of the results

648 In this section, the inferences and patterns that were identified after visually analyzing the results 649 of ResMem-Net are discussed. To aid us with this process, GradRAM technique was used to 650 understand which part of the images are focused by ResMem-Net or in other words, which part 651 of the image gives larger activations. GradRAM is an extension of Class Activation Maps 652 (CAM), which uses the gradient obtained during backpropagation process, to generate heatmaps. 653 The heatmaps generated shed light on which part of the image enhances the image's 654 memorability. This shows how the hidden layers in the ResNet-50 backbone outputs feature maps and it is this information that has is being used by the LSTM unit to make predictions.

656

657

658

659

660

661

662

663

664

665

666

667

668

669

670

671 Based on the heatmaps and careful manual analysis of the results using randomly selected images for different categories from the Isola et al. dataset, the following inferences are made:

The object in the image contributes more to the memorability score than the scene in which the object is placed. In almost every heatmap, it is observable that the portion of the image containing the main object provides higher activations compared to the rest of the image. Also, images with no objects are predicted to be less memorable compared to images containing objects (both living and non-living). Also, images containing a single central object is seen to be more memorable than images with multiple objects. The average rank correlation of the predicted memorability of the images with a single central object is 0.69 , while the average of the rank correlation of the images without a central object is 0.36 . Also, the presence of humans in the image contributes to a better memorability score. If the human in the image is clearly visible, then the memorability averages at 0.68 , while if the image does not contain any human or object, then the memorability averages at 0.31 .

Using a model pretrained on object classification datasets provide better results and trains faster than using a model pretrained on scene classification datasets (Jing et al., 2016). This can be

Peer) Comput. Sci. reviewing PDF | (CS-2021:05:61139:2:0:NEW 23 Sep 2021) 
672 attributed to the fact that memorability scores are directly related to the presence of objects. 673 Thus, a model whose weights contain information about objects take lesser time to converge to a 674 minimum (Best N, Ott J \& Linstead E J, 2020). Also, image aesthetics does not have much to do 675 with Image memorability. A few images containing content related to violence are not 676 aesthetically good, but the memorability score of the image is high, with an average 677 memorability of 0.61 .

678

679

680

681

682

683

684

685

686

687

688

689

690

691

692

693

694

695

696

697

698

699

700

701

702

703

704

705

706

707

708

700

718

713

714

\section{Conclusions}

Capturing memorable pictures is bit challenging as it requires an enormous amount of creativity. However, just like any other phenomenon in nature, humans' capability to remember certain images more follows a pattern. This paper becomes unique by introducing ResMem-Net, a novel neural network architecture that combines a pretrained deep learning model (ResNet-50) and a LSTM unit. The model was trained using One Cycle Learning Policy which allows the use of cyclic learning rates during training. ResMem-Net has provided a close to human performance on predicting the memorability of an image using LaMem dataset which is the largest publicly available dataset for image memorability. The rank correlation of ResMem-Net is 0.679 , which is extremely close to human accuracy 0.68 . This obtained result $6.09 \%$ increase the performance of MemNet, 35\% increase from CNN-MTLES, $2 \%$ increase from MCDRNet and a $1.2 \%$ increase from EMNet. Based on the qualitative analysis executed using GradRAM method, it was inferred that the object plays a bigger role in enhancing the memorability of the image. A pre-trained model that consists of weights from an object classification dataset converges quickly than a model pre-trained on scene classification. These results were observed manually by looking through the highly rated images and lowly rated images. Heatmaps generated using the GradRAM method was also used to analyze and obtain the above inferences.

The limitation of the current work is that even though the model contains much lesser number of parameters than other state-of-the-art models, ResMem-Net is still not deployable to mobile based GPUs. To solve this issue, further research can be done to use mobile compute efficient architectures like MobileNetV3 or EfficientNet, which are also pre-trained on the ImageNet dataset. Further research can also be done to improve the accuracy of the model by replacing ResNet-50 with more recent architectures like ResNext. The LSTM unit can also be replaced with more recent architectures like the Transformer architecture or BiDirectional RNNs. A more generic suggestion is to spend time to develop larger datasets for image memorability prediction because with larger datasets, neural networks can generalize better.

\section{Acknowledgements}

The authors would like to thank the Deanship of Scientific Research at Umm AlQura University for supporting this work by Grant Code: 19- ENG-1-01-0015 
715 1. Kong S, Shen X, Lin Z, Mech R, Fowlkes C. 2016. Photo aesthetics ranking network with attributes

716 and content adaptation. European Conference on Computer Vision 2016 DOI: 10.1007/978-3-319-

717 46448-0_40.

718 2. Celikkale B, Erdem A, Erdem E. 2015. Predicting memorability of images using attention-driven

719 spatial pooling and image semantics. Image and vision Computing 42(C): 35-46 DOI:

720 10.1016/j.imavis.2015.07.001.

721 3. Bainbridge W A, Dilks D D, Oliva, A. 2017. Memorability: A stimulus-driven perceptual neural

722 signature distinctive from memory. NeuroImage 149: 141-152 DOI: 10.1016/j.neuroimage.2017.01.063.

723 4. Schurgin M W. 2018. Visual memory, the long and the short of it: A review of visual working memory

724 and long-term memory. Attention, Perception, \& Psychophysics 80: 1035-1056 DOI: 10.3758/s13414-

725 018-1522-y.

726 5. Gretz M R \& Huff M J. 2020. Multiple species of distinctiveness in memory? Comparing encoding

727 versus statistical distinctiveness on recognition. Memory 28: $984-997$ DOI:

$72810.1080 / 09658211.2020 .1803916$.

729 6. Rust N C, Mehrpour V. 2020. Understanding image memorability. Trends in cognitive sciences 24:

730 557-568 DOI: 10.1016/j.tics.2020.04.001.

731 7. Alves H, Högden F, Gast A, Aust F, Unkelbach C. (2020). Attitudes from mere co-occurrences are

732 guided by differentiation. Journal of personality and social psychology 119(3): 560-581 DOI:

733 10.1037/pspa0000193.

734 8. Fukuda K, Vogel E K. 2019. Visual short-term memory capacity predicts the "bandwidth" of visual

735 long-term memory encoding. Memory \& cognition 47: 1481-1497 DOI: 10.3758/s13421-019-00954-0.

736 9. Isola P, Parikh D, Torralba A, Oliva A. 2011. Understanding the intrinsic memorability of images.

737 Proceedings of the 24th International Conference on Neural Information Processing Systems.

738 10. Isola P, Xiao J, Torralba A, Oliva A. 2011. What makes an image memorable?. IEEE Conference on

739 Computer Vision and Pattern Recognition

740 11. Broers N \& Busch N. (2021). The effect of intrinsic image memorability on recollection and

741 familiarity. Memory \& cognition 49: 998-1018 DOI: 10.3758/s13421-020-01105-6

742 12. Bainbridge W A. 2020. The resiliency of image memorability: A predictor of memory separate from

743 attention and priming. Neuropsychologia 141 DOI: 10.1016/j.neuropsychologia.2020.107408.

744 13. Sommer V R, Mount L, Weigelt S, Werkle-Bergner M, Sander M C. 2021. Memory specificity is

745 linked to repetition effects in event-related potentials across the lifespan. Developmental cognitive

746 neuroscience 48: 100926 DOI: 10.1016/j.den.2021.100926..

747 14. Khosla A, Raju A S, Torralba A, Oliva A. 2015. Understanding and predicting image memorability at

748 a large scale. Proceedings of the IEEE International Conference on Computer Vision DOI:

749 https://doi.org/10.1109/ICCV.2015.275.

750 15. Perera S, Tal A, Zelnik-Manor L. (2019). Is Image Memorability Prediction Solved?. In Proceedings

751 of the IEEE/CVF Conference on Computer Vision and Pattern Recognition Workshops (pp. 0-0).

752 16. Fajtl J, Argyriou, V, Monekosso D, Remagnino P. 2018. Amnet: Memorability estimation with

753 attention. In Proceedings of the IEEE Conference on Computer Vision and Pattern Recognition (pp.

754 6363-6372).

755 17. Squalli-Houssaini H, Duong N Q, Gwenaëlle M, Demarty C H. 2018. Deep learning for predicting

756 image memorability. In 2018 IEEE International Conference on Acoustics, Speech and Signal Processing

757 2371-2375 DOI: 1109/ICASSP.2018.8462292. 
758

759

760

761

762

763

764

765

766

767

768

769

770

771

772

773

774

775

776

777

778

779

780

781

782

783

784

785

786

787

788

789

790

791

792

793

794

795

796

797

798

799

800

801

18. Canchumuni S W A, Emerick A A, Pacheco M A C. 2019. Towards a robust parameterization for conditioning facies models using deep variational autoencoders and ensemble smoother. Computers \& Geosciences, 128:87-102 DOI: 10.1016/j.cageo.2019.04.006

19. Lu J, Xu M, Yang R, Wang Z. 2020. Understanding and predicting the memorability of outdoor natural scenes. IEEE Transactions on Image Processing 29:4927-4941 DOI: 10.1109/TIP.2020.2975957. 20. Baveye Y, Cohendet R, Perreira Da Silva M, Le Callet P. (2016, October). Deep learning for image memorability prediction: The emotional bias. In Proceedings of the 24th ACM international conference on Multimedia 491-495 DOI: 10.1145/2964284.2967269.

21. Mancas M, Le Meur O. 2013. Memorability of natural scenes: The role of attention. In 2013 IEEE International Conference on Image Processing 196-200. DOI: 10.1109/ICIP.2013.6738041.

22. Zhu T, Zhu F, Zhu H, Li L. 2020. Aesthetics-Assisted Multi-task Learning with Attention for Image Memorability Prediction. 2020 IEEE Conference on Multimedia Information Processing and Retrieval 360-363 DOI: 10.1109/MIPR49039.2020.00079

23. Zhao T, Fang I, Kim J, Friedland G. 2021. Multi -modal Ensemble Models for Predicting Video Memorability. arXiv:2102.01173. https://arxiv.org/abs/2102.01173

24. Leyva R and Sanchez V. 2021. Video Memorability Prediction Via Late Fusion Of Deep Multi-Modal Features. 2021 IEEE International Conference on Image Processing (ICIP) 2488-2492 DOI:

10.1109/ICIP42928.2021.9506411.

25. Ellahi W, Vigier T, Le Callet P. 2020. Can Visual Scanpath Reveal Personal Image Memorability? Investigation of HMM Tools for Gaze Patterns Analysis. 2020 Twelfth International Conference on Quality of Multimedia Experience 1-4 DOI: 10.1109/QoMEX48832.2020.9123102 .

26 Basavaraju S, Sur A. (2019). Multiple instance learning based deep CNN for image memorability prediction. Multimedia Tools and Applications 78(24):35511-35535 DOI: 10.1007/s11042-019-08202-y.

27. Selvaraju R R, Cogswell M, Das A, Vedantam R, Parikh D, Batra D. 2017. Grad-cam: Visual explanations from deep networks via gradient-based localization 2017 Proceedings of the IEEE international conference on computer vision 618-626 DOI: 10.1109/ICCV.2017.74.

28. He K, Zhang X, Ren S, Sun J. 2015. Delving deep into rectifiers: Surpassing human-level performance on imagenet classification. In 2015 Proceedings of the IEEE international conference on computer vision 1026-1034 DOI: 10.1109/ICCV.2015.123.

29. Cho K, Van Merriënboer B, Gulcehre C, Bahdanau D, Bougares F, Schwenk H, Bengio Y. 2014. Learning phrase representations using RNN encoder-decoder for statistical machine translation. arXiv preprint arXiv: 1406.1078 .

30. Greff K, Srivastava R K, Koutník J, Steunebrink B R, Schmidhuber J. 2016. LSTM: A search space odyssey. IEEE transactions on neural networks and learning systems 28(10):2222-2232 DOI: 10.1109/TNNLS.2016.2582924.

31. Hsiao T Y, Chang Y C, Chou H H, Chiu C T. 2019. Filter-based deep-compression with global average pooling for convolutional networks. Journal of Systems Architecture 95:9-18 DOI: 10.1016/j.sysarc.2019.02.008.

32. Yi D, Ahn J, Ji S. 2020. An Effective Optimization Method for Machine Learning Based on ADAM. Applied Sciences, 10(3): 1073 DOI: 10.3390/app10031073.

33. Smith L N. 2018. A disciplined approach to neural network hyper-parameters: Part 1--learning rate, batch size, momentum, and weight decay. ArXiv:1803.09820.

34. Rusu A A, Rabinowitz N C, Desjardins G, Soyer H, Kirkpatrick J, Kavukcuoglu K, Pascanu R, Hasell R. 2016. Progressive neural networks. arXiv: abs/1606.04671.

PeerJ Comput. Sci. reviewing PDF | (CS-2021:05:61139:2:0:NEW 23 Sep 2021) 
802 35. Peng H, Li K, Li B, Ling H, Xiong W, Hu W. 2015. Predicting image memorability by multi-view

803 adaptive regression. Proceedings of the 23rd ACM international conference on multimedia DOI:

$80410.1145 / 2733373.2806303$

805 36. Khosla A, Xiao J, Torralba A, Oliva A. 2012. Memorability of image regions. Advances in neural 806 information processing systems 25: 296-304

807 37. Jing, P, Su Y, Nie L, Gu H. 2016. Predicting image memorability through adaptive transfer learning 808 from external sources. IEEE Transactions on Multimedia 19(5):1050-1062 DOI: 809 10.1109/TMM.2016.2644866.

810 38. Best N, Ott J \& Linstead E J. 2020. Exploring the efficacy of transfer learning in mining image-based 811 software artifacts. J Big Data 759 DOI: https://doi.org/10.1186/s40537-020-00335-4 


\section{Table $\mathbf{1}$ (on next page)}

Performance of various architectures on Lamem dataset

$\rho$ values compared 
1

\begin{tabular}{|l|l|}
\hline Method (LaMem dataset) & $\mathbf{( \rho )}$ \\
\hline ResMem-Net (proposed model) & 0.679 \\
\hline MemNet & 0.640 \\
\hline MCDRNet & 0.663 \\
\hline EMNet & 0.671 \\
\hline CNN-MTLES (different dataset split) & 0.5025 \\
\hline
\end{tabular}


Table 2 (on next page)

Performance of various architectures on Isola et al dataset

$\rho$ values compared with another dataset 
1

\begin{tabular}{|l|l|}
\hline Method (Isola et al dataset) & (p) \\
\hline ResMem-Net (proposed model) & 0.673 \\
\hline MemNet & 0.61 \\
\hline MCDRNet & 0.638 \\
\hline EMNet & 0.664 \\
\hline SVR & 0.462 \\
\hline
\end{tabular}




\section{Table 3(on next page)}

Comparison of predicted scores and ground truth scores for various sets of images from the LaMem dataset.

Comparison table between different networks with the proposed 


\begin{tabular}{|l|l|l|l|l|l|}
\hline Dataset & MemNet & MCDRNet & EMNet & ResMem-Net & Ground Truth \\
\hline Top 10 & $91.70 \%$ & $93.15 \%$ & $91.89 \%$ & $93.82 \%$ & $100 \%$ \\
\hline Top 25 & $90.40 \%$ & $91.76 \%$ & $91.36 \%$ & $92.8 \%$ & $100 \%$ \\
\hline Top 50 & $89.57 \%$ & $91.27 \%$ & $90.85 \%$ & $90.52 \%$ & $99.35 \%$ \\
\hline Top 100 & $89.17 \%$ & $90.42 \%$ & $90.38 \%$ & $90.74 \%$ & $98.45 \%$ \\
\hline Top 200 & $88.91 \%$ & $90.00 \%$ & $90.02 \%$ & $90.65 \%$ & $97.57 \%$ \\
\hline Bottom 200 & $55.06 \%$ & $54.57 \%$ & $54.23 \%$ & $52.18 \%$ & $42.16 \%$ \\
\hline Bottom 100 & $54.35 \%$ & $52.79 \%$ & $52.74 \%$ & $49.13 \%$ & $39.01 \%$ \\
\hline Bottom 50 & $54.20 \%$ & $51.65 \%$ & $51.25 \%$ & $48.72 \%$ & $36.3 \%$ \\
\hline Bottom 25 & $54.44 \%$ & $51.20 \%$ & $50.34 \%$ & $48.27 \%$ & $34.41 \%$ \\
\hline Bottom 10 & $58.06 \%$ & $50.94 \%$ & $48.41 \%$ & $47.9 \%$ & $33.57 \%$ \\
\hline
\end{tabular}

1 


\section{Table 4 (on next page)}

Comparison of predicted scores and ground truth scores for various sets of images from the Isola et al. Dataset

Comparison of different networks with the proposed one with another dataset 


\begin{tabular}{|l|l|l|l|l|l|}
\hline Dataset & MemNet & MCDRNet & EMNet & ResMem-Net & Ground Truth \\
\hline Top 10 & $80.16 \%$ & $81.75 \%$ & $82.43 \%$ & $82.61 \%$ & $96.54 \%$ \\
\hline Top 25 & $75.46 \%$ & $79.77 \%$ & $81.41 \%$ & $82.14 \%$ & $94.39 \%$ \\
\hline Top 50 & $75.13 \%$ & $78.57 \%$ & $79.48 \%$ & $80.27 \%$ & $92.24 \%$ \\
\hline Top 100 & $74.32 \%$ & $76.64 \%$ & $77.63 \%$ & $79.76 \%$ & $89.59 \%$ \\
\hline Top 200 & $73.58 \%$ & $74.9 \%$ & $76.64 \%$ & $77.92 \%$ & $85.33 \%$ \\
\hline Bottom 200 & $35.91 \%$ & $34.83 \%$ & $34.08 \%$ & $35.95 \%$ & $22.85 \%$ \\
\hline Bottom 100 & $32.8 \%$ & $31.64 \%$ & $31.66 \%$ & $31.4 \%$ & $18.66 \%$ \\
\hline Bottom 50 & $30.14 \%$ & $29.41 \%$ & $28.94 \%$ & $29.67 \%$ & $14.93 \%$ \\
\hline Bottom 25 & $28.81 \%$ & $26.6 \%$ & $29.47 \%$ & $29.23 \%$ & $10.95 \%$ \\
\hline Bottom 10 & $28.29 \%$ & $26.52 \%$ & $26.86 \%$ & $27.42 \%$ & $5.69 \%$ \\
\hline
\end{tabular}

1 


\section{Table 5 (on next page)}

Comparison of accuracy of ResMem-Net and other traditional machine learning methods on the Isola et al. Dataset

Comparison with traditional machine learning algorithms 
1

\begin{tabular}{|l|l|}
\hline Method & $(\rho)$ \\
\hline Isola et al. & 0.46 \\
\hline Khosla et al. (b) & 0.50 \\
\hline Mancas and Meur & 0.479 \\
\hline Peng et al. & 0.52 \\
\hline ResMem-Net & 0.673 \\
\hline
\end{tabular}




\section{Table 6(on next page)}

Computational Complexity Analysis of proposed and existing models

Computation complexities comparison 


\begin{tabular}{|l|l|}
\hline Model & No. of parameters \\
\hline ResMem-Net & 26 million $\approx$. \\
\hline MemNet & 60 million $\approx$. \\
\hline MCDRNet & 276 million $\approx$. \\
\hline EMNet & 414 million $\approx$. \\
\hline
\end{tabular}

1 
Figure 1

Schematic for ResMem-Net

Schematic diagram of the proposed architecture 


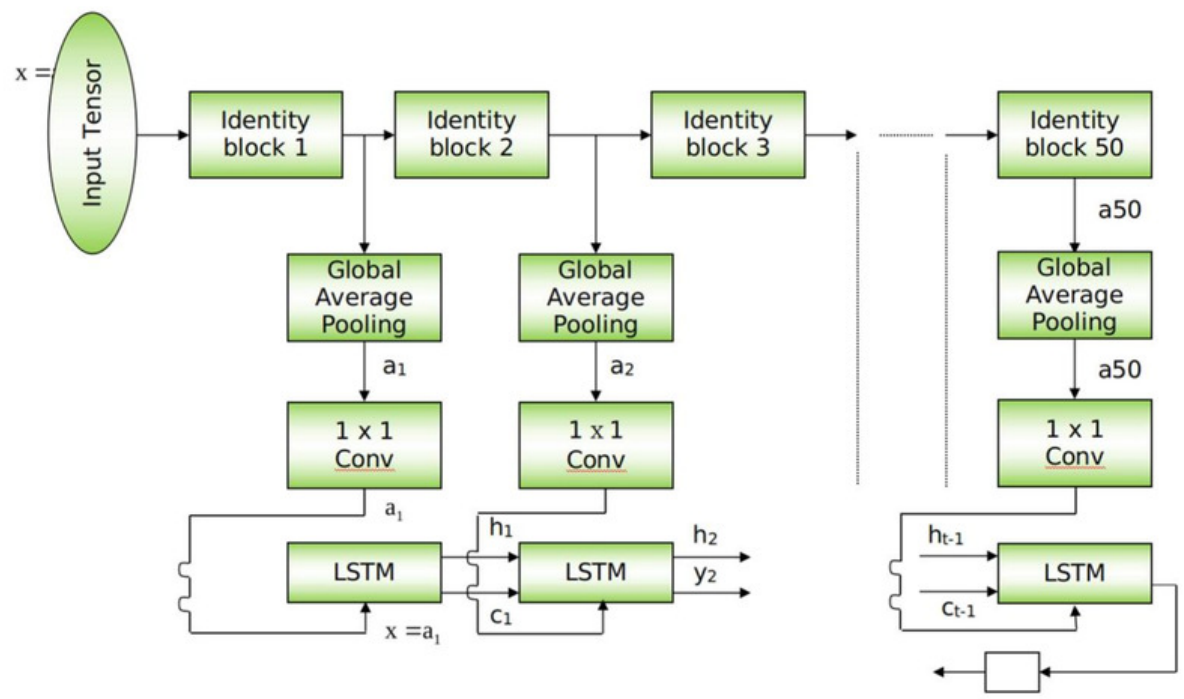




\section{Figure 2}

Flow of data from an intermediate layer of Resnet50 to the LSTM

Flow chart of intermediate layer function 


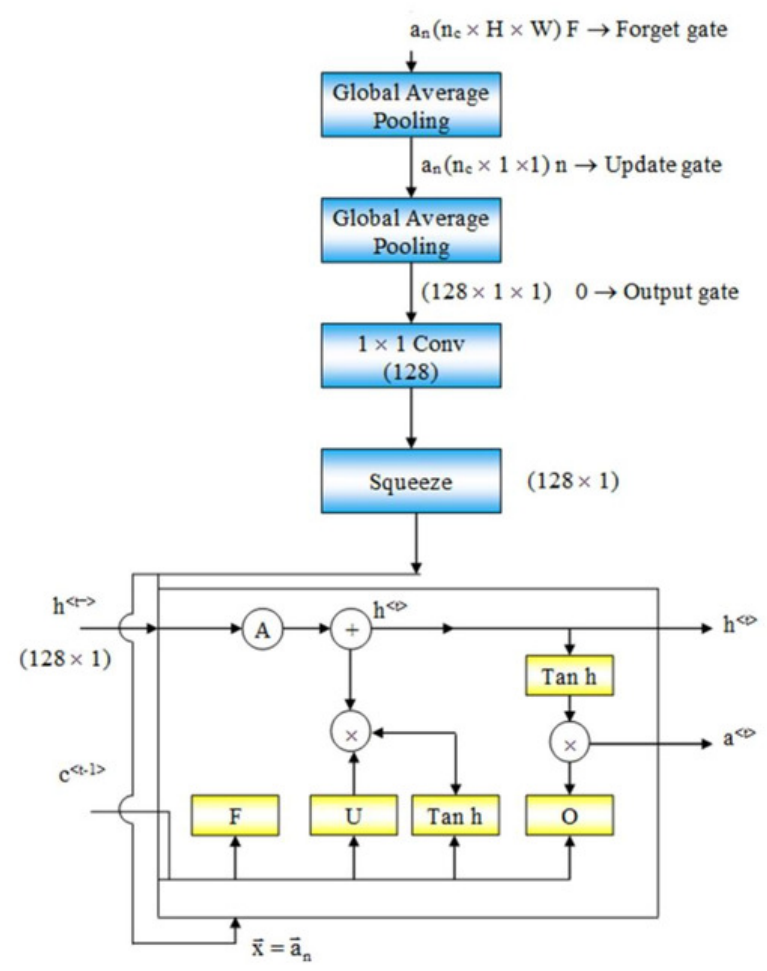


Figure 3

Pipeline used in this work

Pipeline illustration 


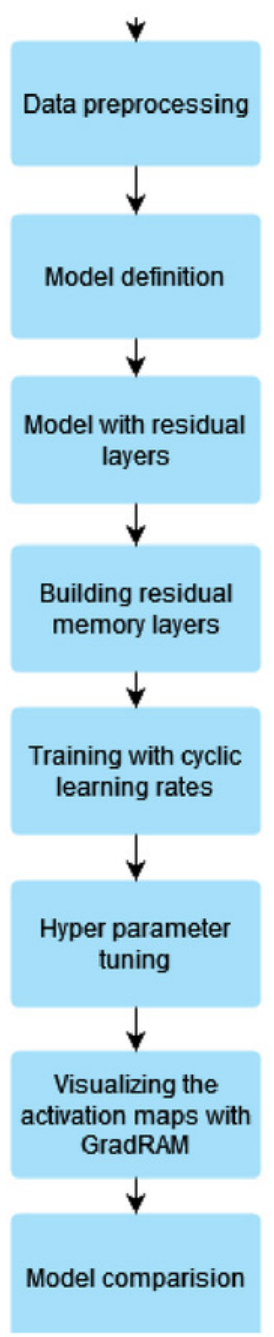




\section{Figure 4}

Bar chart showing the superiority of ResMem-Net over previous works on the LaMem dataset. The results shown are the average of the rank correlation obtained across the validation splits.

Bar chart comparison with first dataset 


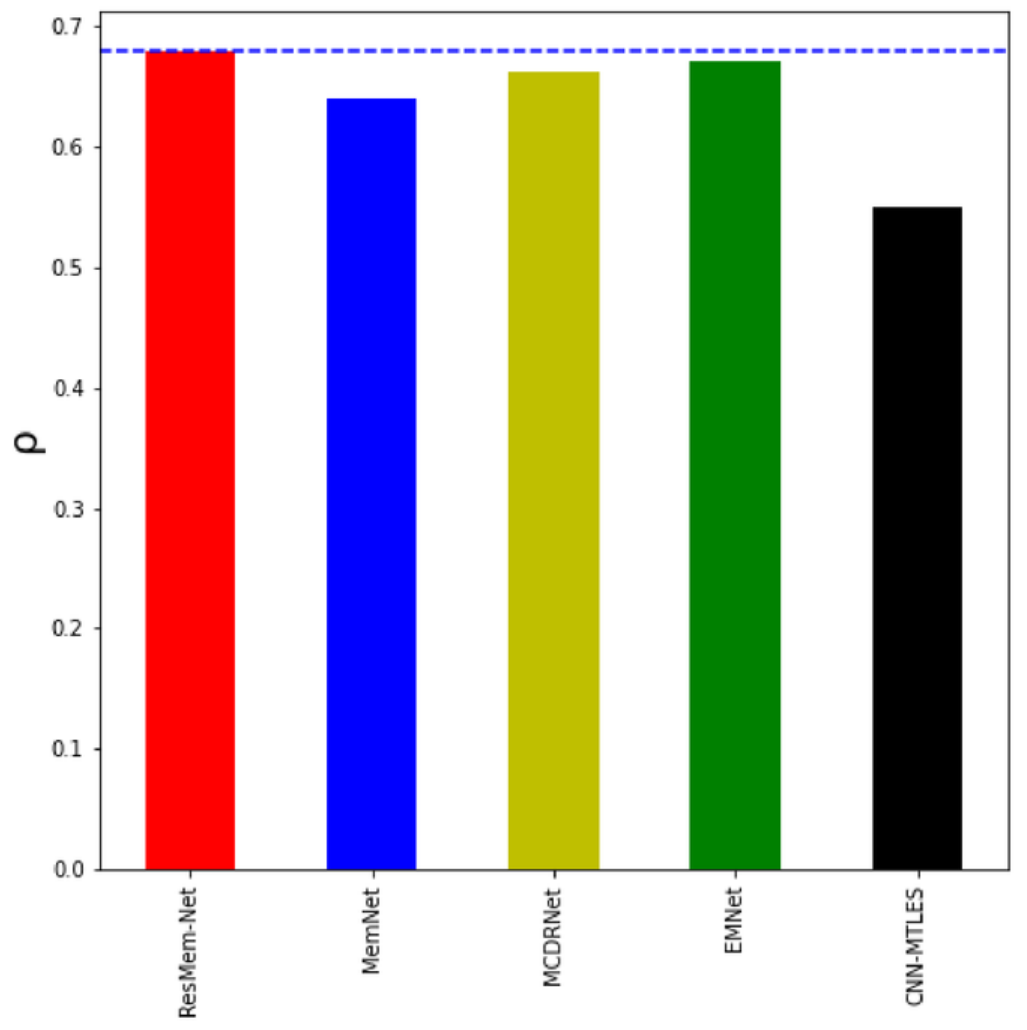




\section{Figure 5}

Bar chart showing the superiority of ResMem-Net over previous works on the Isola et al. dataset. The results shown are the average of the rank correlation obtained across the validation splits.

Bar chart comparison with another dataset 


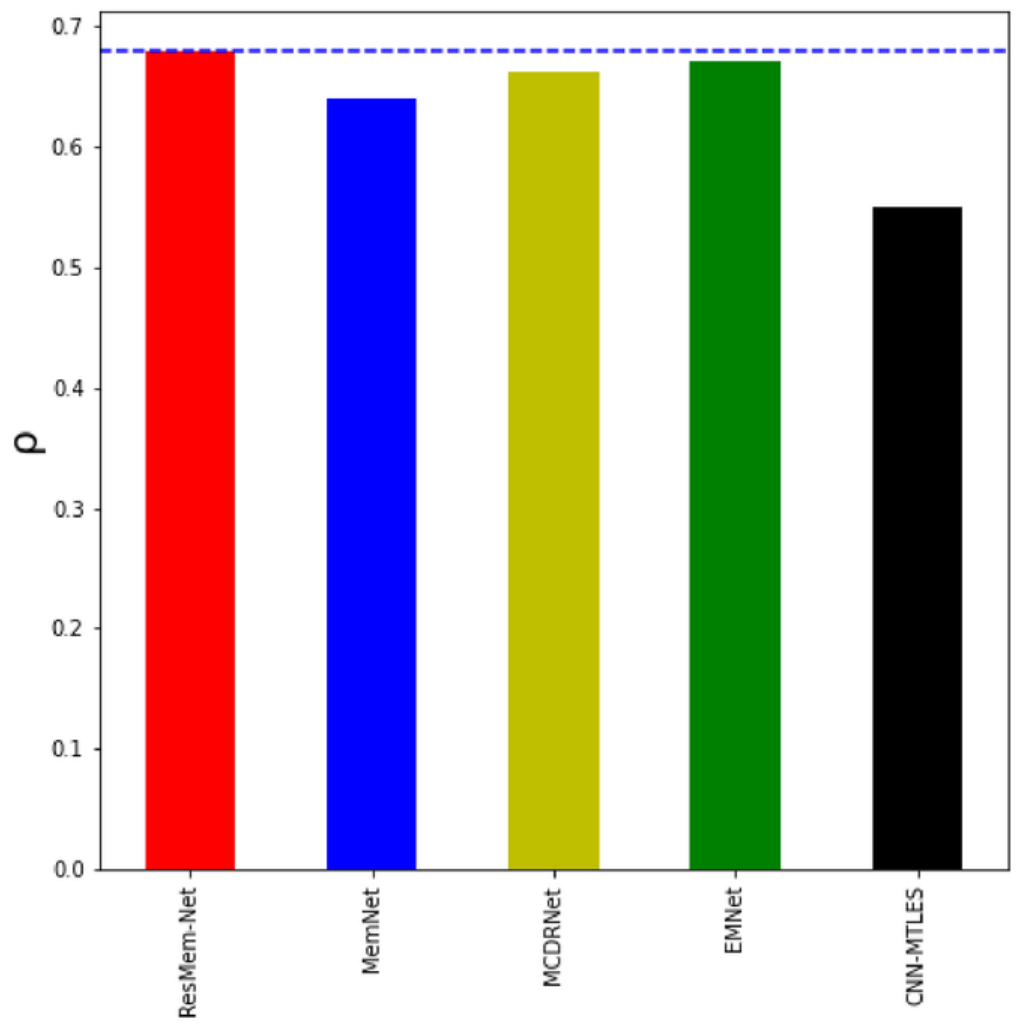




\section{Figure 6}

Bar chart showing the accuracy of non-deep learning based models. The rank correlation values shown are the average of the rank correlation obtained across the validation splits.

Barchart comparison 


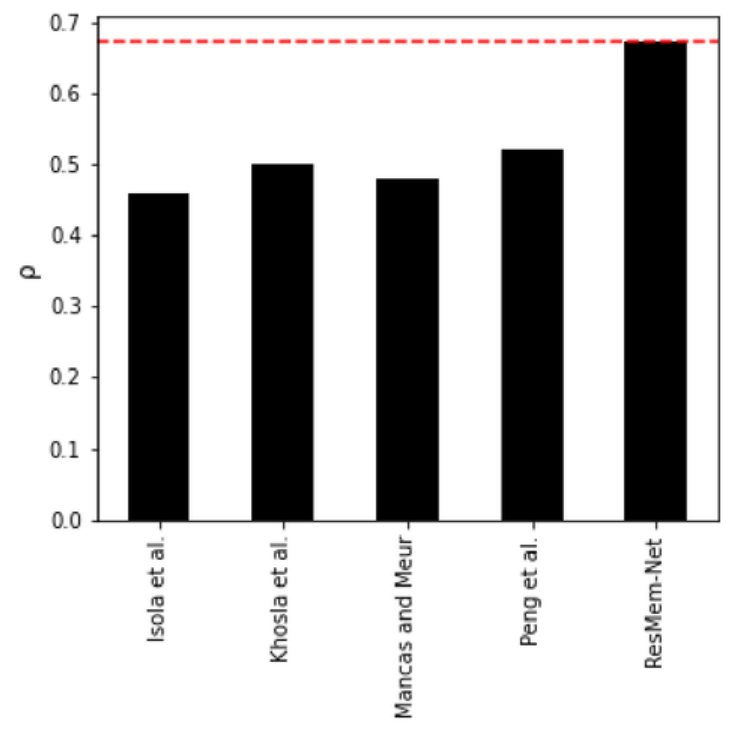

